\title{
Workflow Based Prototype for Criminal Investigation in Bulgaria
}

\author{
George Pashev, Silvia Gaftandzhieva \\ University of Plovdiv "Paisii Hilendarski”, "Tsar Asen" 24, Plovdiv, Bulgaria
}

\begin{abstract}
The basic workflows in the field of Criminal Investigations in Bulgaria which are incorporated in a proprietary information system are presented in this paper. Leading role in the investigation is the prosecutor, but in other countries, this role is more or less consistent with the role of the investigator. EMS monitors the operation of the process and suggests gaps in the work of the officials.
\end{abstract}

Keywords - Workflow, criminal investigation, prosecution.

\section{Introduction}

In the field of criminal investigation in Bulgaria, there is a specific legal framework. This legislation includes, for example, the Penal Code. In the Constitution of the Republic of Bulgaria, the prosecution is part of the judiciary, subordinate to the Supreme Judicial Council [1], [2], [3]. Therefore, a full-fledged investigation is carried out by representatives of various authorities: on the one hand, the prosecution in the judiciary, on the other hand, investigating police officers in the executive branch, and in particular the Ministry of the Interior.

This complex distribution of investigative bodies, as well as the wide variety of investigative activities and processes, requires the use of a modern approach

DOI: 10.18421/TEM101-44

https://doi.org/10.18421/TEM101-44

Corresponding author: George Pashev, University of Plovdiv "Paisii Hilendarski". Email: georgepashev@uni-plovdiv.bg

Received: 15 September 2020.

Revised: 28 December 2020.

Accepted: 05 January 2021.

Published: 27 February 2021.

(c) BY-NC-ND(C) 2021 George Pashev \& Silvia Gaftandzhieva; published by UIKTEN. This work is licensed under the Creative Commons AttributionNonCommercial-NoDerivs 4.0 License.

The article is published with Open Access at www.temjournal.com to investigative records like these, described in [4], [5].

Such an approach would make use of workflow systems and frameworks, as well systems which would allow complex development (G1) and adaptability like [6], [7], [8].

Wen, Man, Le \& Shi [9], focus on cloud development and the use of Software as a Service (SaaS). Some of the reviewed approaches make use of technologies in the field of Machine Learning and Artificial Intelligence like [10], [11]. Some very specialized approaches in the field of computer crime investigation in Microsoft Windows environment are discussed in [12].

In Balkan countries such as Bulgaria, the legal framework is very variable and depends on many factors including political and economic ones [13].

For this reason, software systems have to allow maximum adaptability to rapidly changing legal realities (G2). Therefore, the use of workflows is necessary (G3). But in addition to this requirement, other requirements have to be taken into account, such as the ability of the system to work with clients while developers develop new versions (continuous operation) (G4), maximum preservation of old data from old files, which were developed under the old legal framework (G5). This would to some extent impose limits when using Machine Learning.

The use of different approaches and environments to work with semi-structured data (G5) such as NoSQL [14] engines are more adequate than the use of highly structured and interconnected data paradigms such as SQL.

The paper aims to present a software prototype of a software system that meets the requirements mentioned above.

\section{Description of the Processes and Considerations for the Synthesis of Definitions of Processes}

To meet our Goals (G1-G5), we created several data classes, steps, processes (including processes, sub-processes and a midstream process) by using EMS. As a result, our "Prosecution prototype" (EMSPP) emerged.

Some of these processes in EMS-PP are discussed below. 


\subsection{Sending Out and Receiving Back the File - 07}

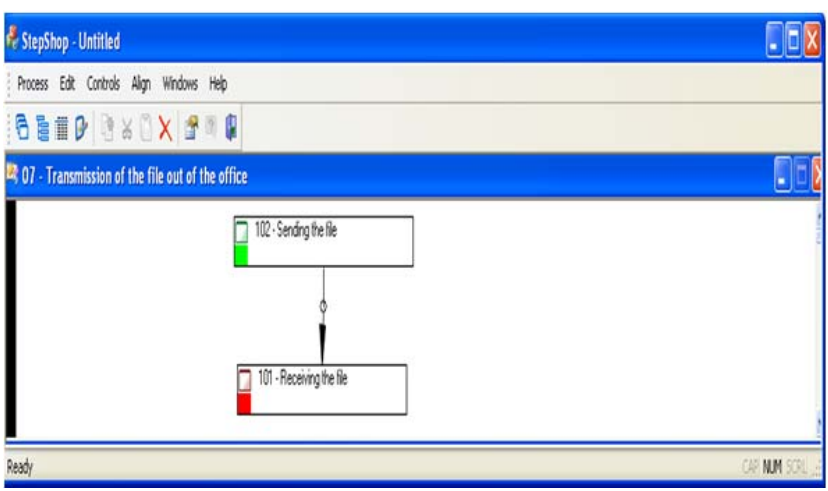

Figure 1. File transmission process

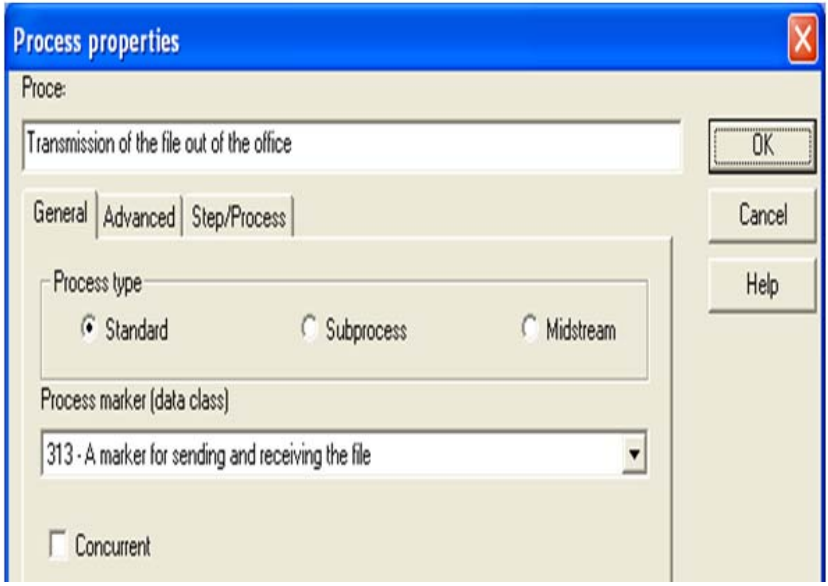

Figure 2. File transmission process properties

This process (shown in Fig. 1) involves only two events/steps: 102 Sending the file; 101 Receiving the file. The parameters are shown in Fig. 2;

The reason for defining such a short process is the relationship between sending and receiving. Rules for keeping the papers require that sending the file has to have resulted in a return of the file. The officer has a task to monitor compliance with this complexity. The user's screen, this process is visualized as a finished or unfinished.

The process is marked/tagged with field class 313 Marker for sending and receiving the file. This field establishes the reason for sending the file to a free and understandable form. This data have to be understandable to the employees.

The process is of type Standard. This allows the process to be added at any point in the global business flow. This reflects the fact that there are no procedural barriers to the file to be sent out at any time.

\subsection{Process of Imposing a Supervision Measure of a Person-04}

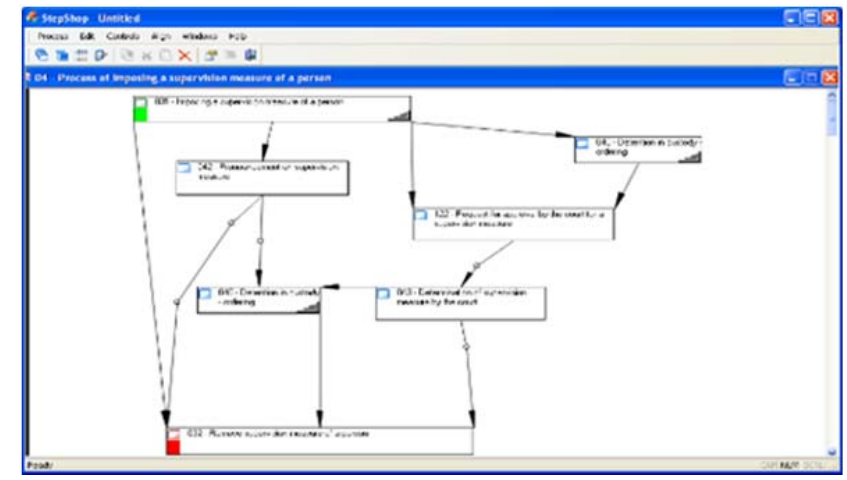

Figure 3. Supervision measure process definition

Fig. 3 depicts several steps 7 . The sequence is displayed with arrows.

The arrangement of the elements of the process with a graphical tool and formation parameters of the process are introduced in the same tool (EMS StepShop). More complex interdependencies can be programmed triggers.

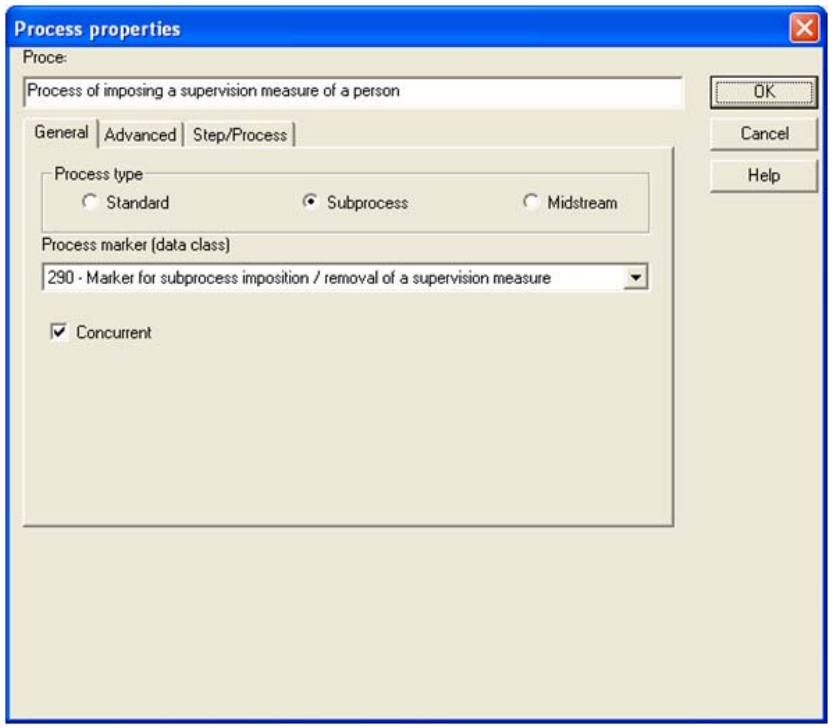

Figure 4. Imposing a supervision process properties

Two-way arrows foresee the existence of the two tiers.

Two steps in Fig. 3 contain in the lower right corner a symbol, which indicates that these steps have satellites. With all of these steps, other related steps may be present. This is a simple form of hierarchical nesting.

For the process presented in Fig. 4 two parameters are chosen. These parameters indicate the interaction of this process to other processes in the global business flow: 
- Sub-process - indicates that this process has to be embedded into another process. The events of this process can occur only within the indictments. Later in the description of the prototype will be clear that the restriction of a person can be taken only under indictment. This process does not describe pre-trial detention for persons detained at the scene.

- Concurrent - indicates that more than one active process may exist simultaneously. There could be measures against several individuals or several measures against a person.

The prototype does not cover all details of the procedural laws, but can be expanded with the following sample steps: Notify family and employer; Notification from the Ministry of Foreign Affairs for detained foreigners; Child care for detainees.

\section{Step 031 - Imposing a supervision measure of a person}

Step 031 is described in Fig. 5.

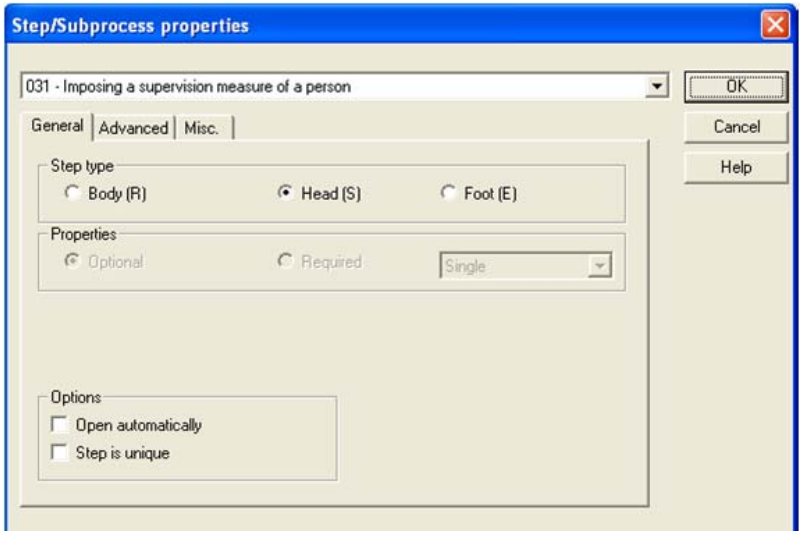

Figure 5. Step properties of step 031

A person with a restriction shall be entered satellite step 006 Person with the charge. Step 031 combined with 006 satellite carries the ground by law, the date, time, type of restriction (Type of measure of procedural coercion). The process is highlighted/ marked with data class 290. It contains a free written description of actions taken. Standard logic of the system allows adding step 031 only if there is an active process in which this sub-process is put. More precisely, only if there is a process to charge a single person, that person can begin a process of this kind (the process "Accusation/ indictment" is described later in this paper).

Step 040 - Detention in custody - ordering This is the order imposing the most severe restrictions as detention. After this step one has to seek approval from the court by step 122 ;

Step 122 - Request for approval by the court for a supervision measure - The approval of the court is sought for different weight restrictions in different countries. Therefore, the inclusion of step 122 may be refined by a trigger. Standard logic step 122 allows adding only if there is an active process of this kind. Additional logic added to a trigger "entry a122" filtered among active processes as required by applicable laws.

Step 122 is defined by a unique process. This implies that one cannot send more than one request to the court.

The prototype does not cover all details of a referral to court, but can be expanded in the following areas:

- Appeal against the decision of the first instance court;

- Appeal against the decision of the second instance court;

- Complaints of the person in front of the upper body of the investigation department.

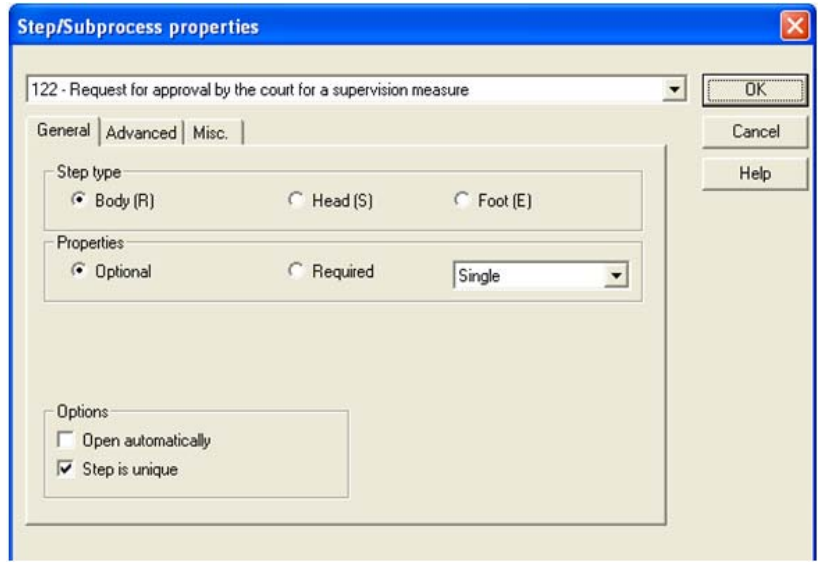

Figure 6. Step properties of step 122

\section{Remove supervision measure of a person}

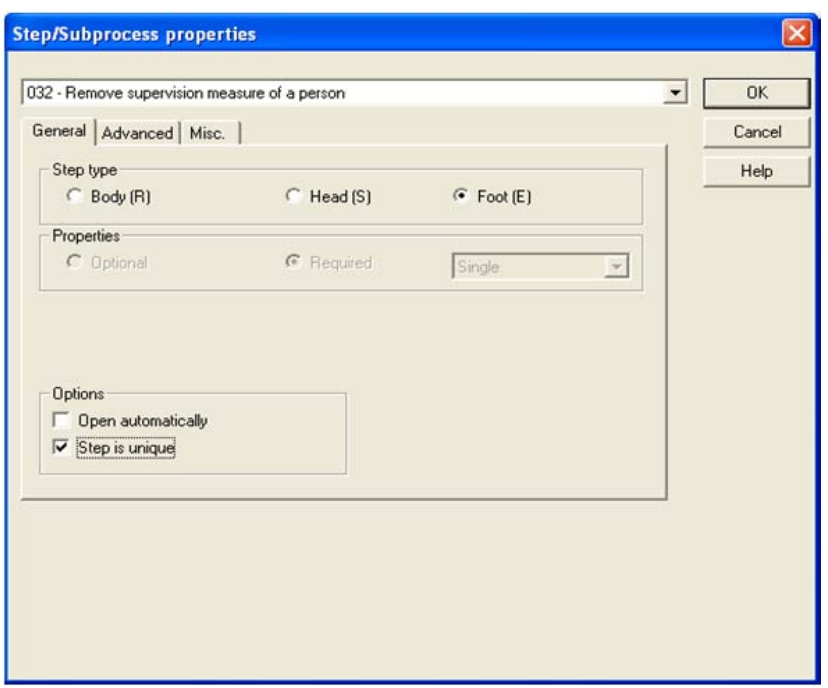

Figure 7. Step properties of step 032

Detention is to be made in advance and then seek permission from the court. Under other conditions, it may start with custody and after judicial authorization to issue a new order for permanent retention. Then it 
should instead step 40 have two separate steps for provisional detention and permanent retention. Then, this process would be complicated, but the principles for implementation of the process are retained.

\section{Step 035 - Permission to visit a detained in custody}

Permission to visit (step 035) is designed only as a satellite of available step 040. This is not shown in the diagram, but only 035 is a satellite 040 and cannot be incorporated in any other way in the process.

\subsection{Assigned Audit / Inspection / Examination 02}

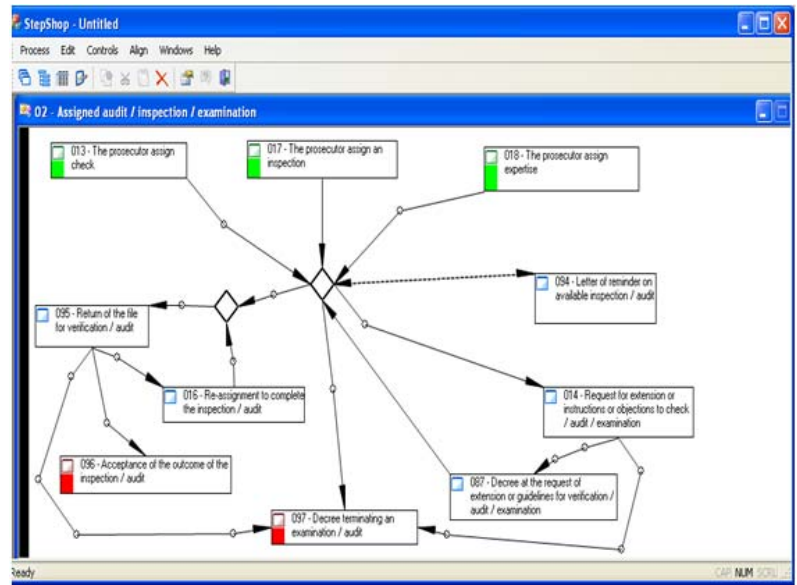

Figure 8. Definition of Process 02

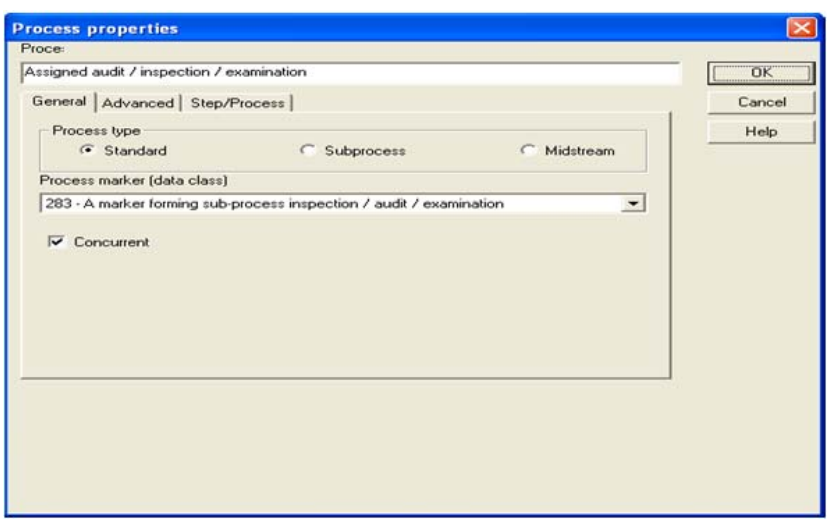

Figure 9. Process properties of process 02

Fig. 8 and Fig. 9 present the basic parameters of the process. In contrast to Fig. 4, the process type Standard is selected. This means that the process cannot exist independently or for the overall business process. Procedurally, this difference in Fig. 4 allows such a process to be performed before an accused person or not associated with an accused person. This approach means that the system will not automatically alert after appropriate charges. A different approach is also possible. The developer could create a process that is introduced and implemented in the indictment. Then one can avoid the mistake of users when the charges were dropped, and the checks continue.
Steps 13, 17 and 18 are alternative possibilities for the start of this process. Fig. 9 depicts this immediately after the start-up steps, such a binding element, i.e. sequence in all three cases is the same. The combination of three different but structurally related procedures in this prototype illustrates the possibility of combining the same routes with other data in the respective steps.

The two steps 95 and 16 allow the arrows to form a cycle. The parameter is allowing it to run more than one time, which is shown in Fig. 10.

Step 95 has two properties: Required and Multiple. This means that the step has to be displayed at least once, but it may occur more than once. For the next step in the cycle (step 16) the parameters are shown in Fig. 12.

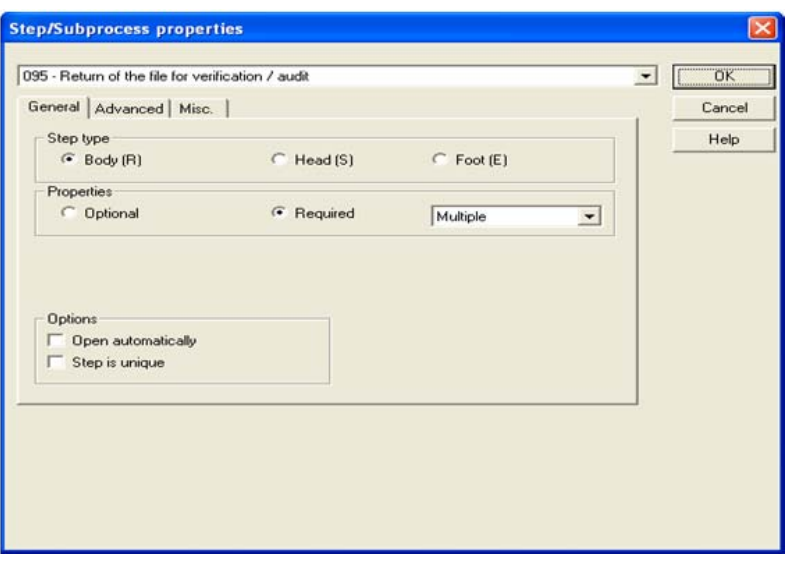

Figure 10. Step properties of Step 095

Apparently, on the screen, the step does not require being compulsory and may be present more than once. Links with arrows serve as parameters for the system. Step 16 has to be followed again with step 95 . To the final step 96 may only pass through step 95 . The final step 97 can be switched at any time, except for the moments when the prosecutor called for the extension through step 87 or assigns complete the check by step 16. More details on defined parameters can be seen by looking at the prototype with StepShop.

\subsection{Accusation / Indictment 03}

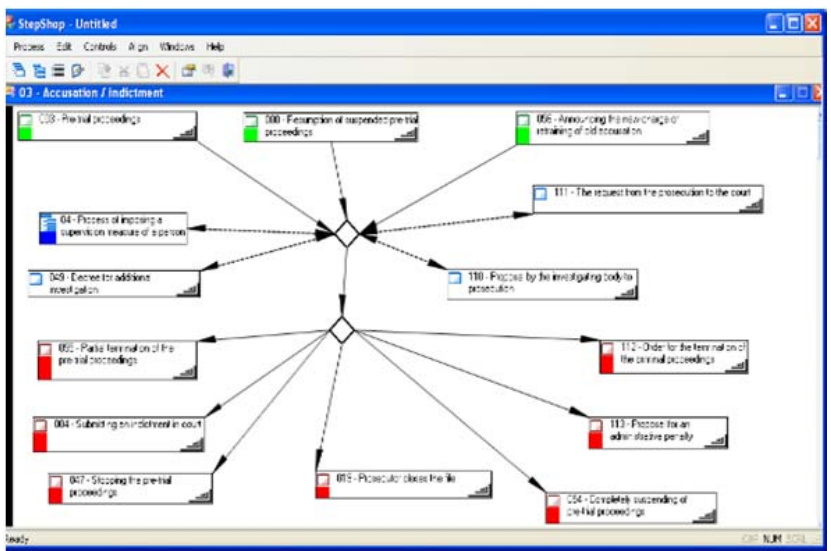

Figure 11. Process definition of process 03 
Each process applies to a convicted person. If a current file shows that the number of charges against a person can become very high, it could be made so that the charges can be grouped for a person to register a manageable number of charges. In the formation process for each crime, it allows the EMS to monitor separately the completion of prosecution for any offense. If there is a change in the legal qualification of an indictment, the EMS can monitor whether the charge becomes heavier or lighter. Then EMS can monitor any change in the gravity of the charges, and to highlight the above authorities and the victims. This can make the administrative control of actions by the prosecutor easier. Similar administrative control can be realized and partially dropout charges. The system can be simplified by bringing only a general accusation of one person. Then the diagram of Fig. 11 will remain the same, but we will observe fewer processes.

The difference from the processes described before is input sub-process 04 Process of imposing a supervision measure of a person (Fig. 12).

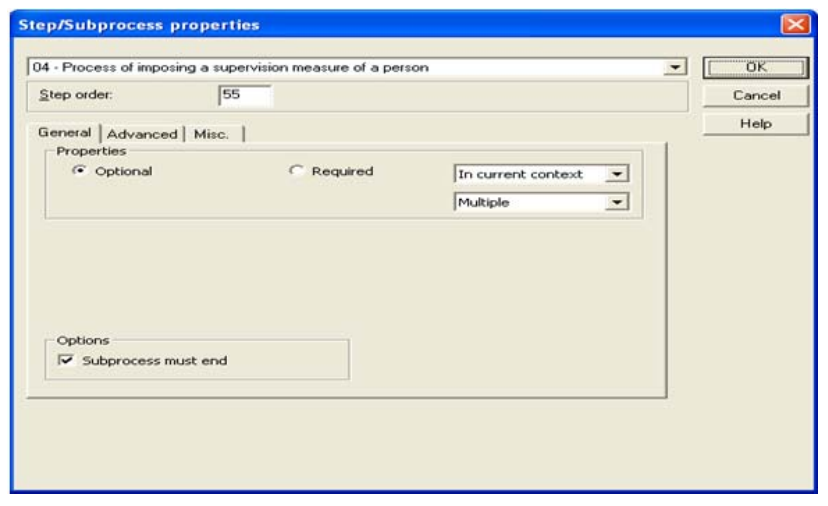

Figure 12. Process properties of process 04

This sub-process is embedded hierarchically with three main parameters:

- Optional Multiple;

- Sub-process has to end.

This means one can have a restriction (detention). Then if the offence is to be discontinued EMS will be signaled to stop detention. In practical realization, the developer has to decide whether the restrictive measure is to be connected to a separate charge for the person or totally of all charges for the person.

\subsection{Require Material or Information 06}

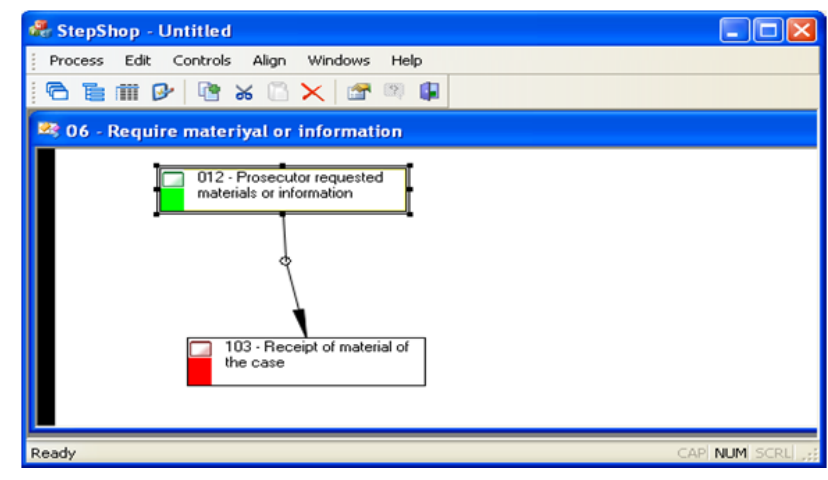

Figure 13. Process definition of process 06

Materials and information are regularly required by public and private entities. Through this process, it is observed whether each of correspondents has provided the requested information. Materials and information are required during all phases of the criminal investigation. The law permits it even before prosecutions.

\subsection{Pre - Processing of Signals or Letters 11}

This process differs significantly from all of the processes described here. It belongs to a group of processes which cannot coexist. This set of processes is called Midstream. Even if several processes are defined with that status, they may not exist at the same time. In criminal papers, it is customary to define different phases of the development in the investigation. Midstream processes are intended to encapsulate the events corresponding to these phases. In such a process steps and other processes can be used. If we have previously defined an appropriate number of processes from the previous two types, then the definition of Midstream is simplified because it contains fewer elements.

Preliminary signal processing or complaint is a trigger to perform several checks. Based on the analysis of the results, the prosecutor may decide to continue the investigation or to suspend or send the file by competence. These requirements are described in the following Figure. 


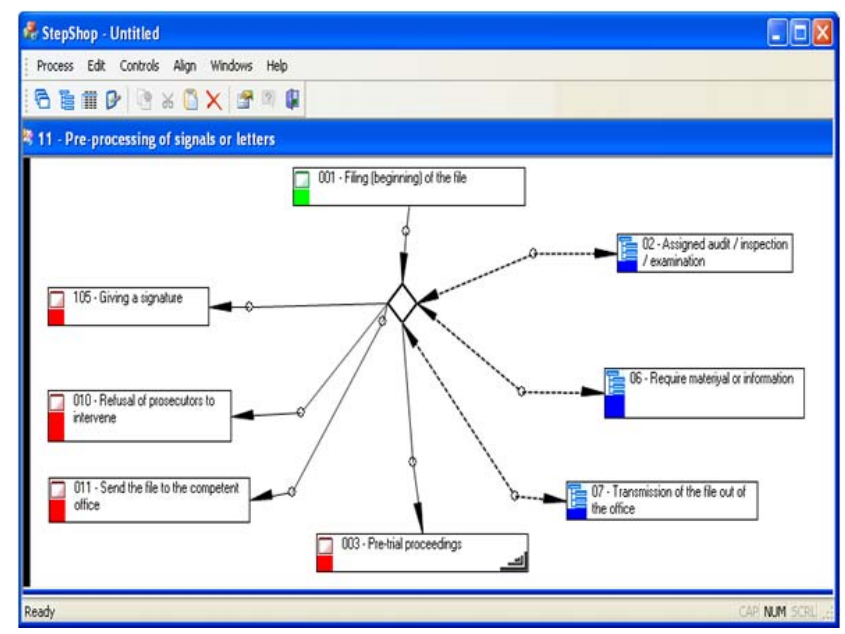

Figure 14. Definition of Process 11

\subsection{Pre - Trial proceedings 12}

All major activities in charge are carried out in this phase, the actual interaction with other institutions as well, and then send as the charge in court. Only one of such a process may happen, and therefore it is described as Midstream. Fig. 15 depicts the relationships that allow other processes to be entered with weak conditions for lineup one after another. Weak requirements for the ordering of events in this process reflect the freedom to perform a variety of proceedings within the current indictments. Freedom to organize is compensated by stronger relationships within each sub. Thus the severity of the conditions of the legal procedures is carried into sub-processes. This is logical because the individual charges or investigations or individual imposed coercive measures follow each of its procedural requirements.

This process is often called a pre-trial phase. In the prototype is not specified ending step because the investigation is completed separately for each charge.

However, it is possible to assume that the transmission of the file to the trial phase completes the process.

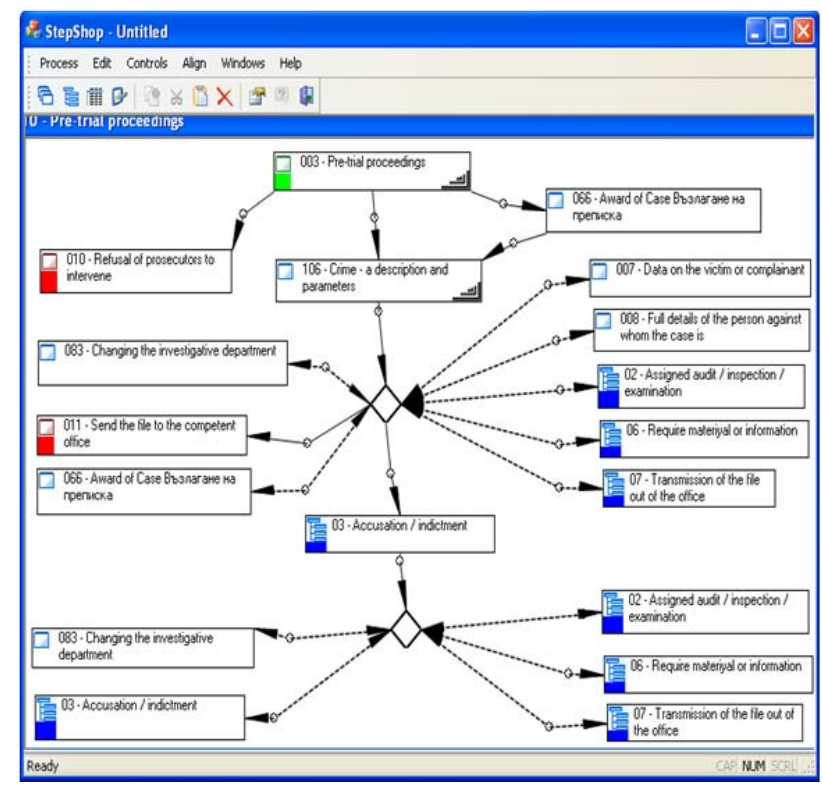

Figure 15. Definition of Pre-trial proceedings Midstream

\section{Conclusion and Future Work}

We managed to achieve our goals (G1-G5) with fewer efforts with the EMS framework and programming paradigm.

Some future work regarding the processes of development has been already discussed in Section 2. Moreover, future legislation changes would result in some development effort to alter the processes, steps and data classes in EMS-PP. In our view, further research is needed on how to make this continuous development more straightforward by the development of intelligent advisors in EMS Step Shop IDE, providing possibilities for data proceedings and performance analysis of the court decisions [15], [16].

We also need tools that could easily transform court decision texts into EMS records (step data) by using text parsing techniques and class data automated discovery by using parser generators for GLR parsers [17] and OCR tools. 


\section{References}

[1]. Marinova, G. (2006). Bulgarian criminal procedure: The new philosophy and issues of approximation. Review of Central and East European Law, 31(1), 45-79.

[2]. Gachevska, K. (2012). Fighting organised crime as a security threat: the lessons learnt from the case of Bulgaria. Journal of Regional Security, 7(1), 63-75.

[3]. Ganev, V. I. (2013). Post-accession hooliganism: Democratic governance in Bulgaria and Romania after 2007. East European Politics and Societies, 27(1), 26-44.

[4]. Zhao, J. L., Bi, H. H., \& Chen, H. (2003, June). Collaborative workflow management for interagency crime analysis. In International Conference on Intelligence and Security Informatics (pp. 266-280). Springer, Berlin, Heidelberg.

[5]. Brown, C. M., Clark, Y., Julian, R., \& Kelty, S. (2018). A step towards improving workflow practices for volume crime investigations: outcomes of a 90day trial in South Australia. Police Practice and research, 19(3), 209-221.

[6]. Prayudi, Y., \& Riadi, I. (2018). Digital Forensics Workflow as A Mapping Model for People, Evidence, and Process in Digital Investigation. International Journal of Cyber-Security and Digital Forensics, 7(3), 294-305.

[7]. Mayer, W., Casanovas, P., Stumptner, M., (2017). SemanticWorkflows in Law Enforcement Investigations and Legal Requirements, in: Proceedings of the 1st Workshop on Technologies for Regulatory Compliance co-located with the 30th International Conference on Legal Knowledge and Information Systems (JURIX 2017).

[8]. Stumptner, M., Mayer, W., Grossmann, G., Liu, J., Li, W., Casanovas, P., ... \& Bainbridge, B. (2015). An architecture for establishing legal semantic workflows in the context of Integrated Law Enforcement. In AI Approaches to the Complexity of Legal Systems (pp. 124-139). Springer, Cham.
[9]. Wen, Y., Man, X., Le, K., \& Shi, W. (2013, May). Forensics-as-a-service (faas): computer forensic workflow management and processing using cloud. In The Fifth International Conferences on Pervasive Patterns and Applications (pp. 1-7).

[10]. Memon, Q. A., \& Mehboob, S. (2003, December). Crime investigation and analysis using neural nets. In 7th International Multi Topic Conference, 2003. INMIC 2003. (pp. 346-350). IEEE.

[11]. Rossy, Q., \& Ribaux, O. (2014). A collaborative approach for incorporating forensic case data into crime investigation using criminal intelligence analysis and visualisation. Science \& Justice, 54(2), 146-153.

[12]. Neil, A. M., Elmogy, M., \& RIAD, A. (2013). A Proposed Framework for Crime Investigation Based On Windows Registry Analysis. Journal of Engineering and Applied Science, Faculty of Engineering, Cairo University, 60(1).

[13]. Siegelbaum, J. H. (2002). The right amount of rights: Calibrating criminal law and procedure in postcommunist Central and Eastern Europe. BU Int'l $L J, 20,73$.

[14]. Han, J., Haihong, E., Le, G., \& Du, J. (2011, October). Survey on NoSQL database. In 2011 6th international conference on pervasive computing and applications (pp. 363-366). IEEE.

[15]. Panayotova, G., Dimitrov, G. P., Petrov, P., \& Os, B. (2016, September). Modeling and data processing of information systems. In 2016 Third International Conference on Artificial Intelligence and Pattern Recognition (AIPR) (pp. 1-5). IEEE.

[16]. Dimitrov, G. P., Panayotova, G., Garvanov, I., Os, B., Petrov, P., \& Angelov, A. (2016, September). Performance analysis of the method for social search of information in university information systems. In 2016 Third International Conference on Artificial Intelligence and Pattern Recognition (AIPR) (pp. 15). IEEE.

[17]. McPeak, S., \& Necula, G. C. (2004, March). Elkhound: A fast, practical GLR parser generator. In International Conference on Compiler Construction (pp. 73-88). Springer, Berlin, Heidelberg. 